\title{
Nuevos escenarios, nuevas propuestas, otras actoras: licenciadas indígenas y la Universidad Veracruzana Intercultural ${ }^{*}$
}

\author{
Inés Olivera Rodríguez \\ Universidad Nacional Autónoma de México \\ inesolivera@gmail.com
}

RESUMEN

El presente artículo trae una discusión en torno a la aparición, en México, de mujeres indígenas licenciadas como resultado de la construcción de un modelo educativo que se pretende cuestionador de la lógica desarrollista imperante. Describe y analiza el papel de la politica de educación superior intercultural como et detonante en la aparición de este perfil profesional, que viene mostrándose eficaz en la generación de transformaciones, familiares, comunitarias y regionales. Parte de una discusión en torno al surgimiento de dicha politica, y los debates en torno a sus limites y posibilidades, y aterriza en la propuesta específica de la Universidad Veracruzana Intercultural (UVI). Para ello analiza las narraciones biográficas de cinco egresadas de la primera generación y la comparación intergeneracional entre una de ellas, su madre, su abuela y su bisabuela, a fin de identificar lo que la experiencia en la UVI aporta a la constitución de estas nuevas actoras.

Palabras clave: educación superior intercultural, profesionales indigenas, mujeres indigenas, interculturalidad, desarrollo.

* Este trabajo presenta los resultados de investigación de la tesis de maestría titulada ¿Desarrollo o buen vivir? Efecto educativo en un contexto intercultural. Experiencias de mujeres indigenas de Huazuntlán - Veracruz, realizada entre agosto de 2011 y diciembre de 2013 en el posgrado de antropología de la UNAM. Agradezco al doctor Gunther Dietz, del IIE-UV, por su acompañamiento constante y encaminamiento en el proceso de investigación, como director de tesis, y al doctor Fernando Nava López, coordinador del posgrado en Antropología de la UNAM en el período de la investigación. Finalmente, agradezco de forma especial la lectura y comentarios de Sergio Hérnandez Loeza. 


\section{New Scenarios, New Proposals, New Female Actors: Indigenous Graduate Women and the Intercultural University of Veracruz}

\section{$A B S T R A C T$}

This article discusses the emergence of new actors among graduate students in Mexico, specifically of indigenous graduate women, who are one of the results of an educational model that intends to question the prevailing developmental logic. It describes and analyzes the role of intercultural higher education policies as catalyst for an emerging professional profile that is having important impacts in building new family, community and regional relations. Through the discussion of these policies, their origins, limits and possibilities, this paper focuses on the specific proposal of the Universidad Veracruzana Intercultural (UVI). It also examines the life experiences of five UVI female graduates, and concentrates in one case in which her trajectory is compared to the life experiences of her own mother, grandmother and great-grandmother. Through these cases, the paper seeks to identify the ways in which the UVI contributes to the constitution of these new female actors.

Keywords: intercultural higher education, indigenous professionals, indigenous women, interculturality, development. 


\section{PALABRAS INICIALES}

Este trabajo basa sus reflexiones en la comprensión del vínculo entre el desarrollo y la educación que estableció la teoría del capital humano a mediados del siglo pasado, definiendo una interpretación de efecto educativo (o razón de ser de la educación formal) que centraliza el incremento de las posibilidades de empleabilidad y acceso a salario (Olivera Rodríguez, 2015). A pesar de los cambios en las condiciones políticas y económicas, acentuados hacia el fin del siglo pasado (Muruyama, 2009), hoy las nociones de empleabilidad y desarrollo (local, regional o nacional) continúan guiando tanto las misiones de los programas educativos, como los criterios de evaluación para el aseguramiento de la calidad (Carot, Castro y Ojeda, 2013).

En este marco, se sostiene aquí que no es posible mirar los nuevos espacios, constituidos con el surgimiento de las Universidades Interculturales (UI), y el consecuente incremento de jóvenes licenciadas indígenas ${ }^{1}$, desde las metas y preocupaciones de la universidad convencional ${ }^{2}$. Estas nuevas actoras, población emergente en México por las dimensiones recientemente alcanzadas, representan un fenómeno de la última década, y confrontan la comprensión convencional de

1 Según datos de la Dirección de Educación Media Superior y Superior de la Coordinación General de Educación Intercultural y Bilingüe de la Secretaría de Educación Pública de 2015, en el ciclo escolar 2015-2016 el 54\% de la matrícula total de las once UI reconocidas por el sistema correspondía a mujeres, y al ver la matrícula por universidad la población femenina puede llegar a representar el 60\% http://eib.sep.gob.mx/diversidad/wp-content/uploads/2016/06/ Matricula-total-15-16-Octubre-2015.pdf

2 Dentro del campo de debate producido en México en torno a la educación superior intercultural, con investigadores internos y externos a las UI, se denomina universidades convencionales a las universidades que no se definen por un enfoque intercultural. Esto evidencia que, aunque en la práctica no todos los académicos vinculados con las UI centralicen el modelo de interculturalidad crítica como lo distintivo de esta propuesta educativa, al definirse en contraposición a una educación convencional, evidencian la importancia de la propuesta pedagógica y éticopolítica de la interculturalidad crítica como lo diferencial. 
efecto educativo al optar por programas universitarios en sus localidades o regiones, donde el uso de la lengua materna es central, donde se incorporan saberes locales, y principalmente, donde la meta es la vinculación comunitaria para el beneficio de las localidades, en formaciones que centralizan el bien colectivo basado en el principio de pertenencia. De esta forma, analizar los efectos de un proyecto educativo como el de la UVI exige una mirada reflexiva, capaz de identificar las particularidades de la propuesta educativa y su valor transformador más allá de la empleabilidad. Estos efectos son pasibles de identificación solo a partir del análisis de percepciones y experiencias de las egresadas.

Ante tal panorama, este trabajo se pregunta: ¿qué caracteriza a estas nuevas actoras, las licenciadas indígenas egresadas de la educación superior intercultural? En otras palabras, ¿qué aportes diferenciales se construyen en la experiencia universitaria de la educación superior intercultural? Las reflexiones que surgen a partir de estas preguntas se alimentan de la información construida en diálogo con cinco egresadas de la primera generación de estudiantes de la Universidad Veracruzana Intercultural (UVI). En cuatro de los cinco casos pude conversar también con las madres de las egresadas, y en uno de estos cuatro casos, las entrevistas se ampliaron a su abuela y su bisabuela.

\section{ACTORAS EMERGENTES EN UN VIEJO PROYECTO DE PROFESIONALIZACIÓN INDÍGENA}

La construcción de una intelectualidad indígena ha sido un largo proceso en la historia republicana mexicana (González Apodaca, 2008). Sin embargo, si bien el siglo XX apostó por una clase profesional indígena, es la creación de las universidades interculturales la política que ha permitido gestar modelos educativos diferentes con posibilidad de construcción de saberes que incluyen a los pueblos indígenas y las formas colectivas y comunitarias de hacer, avanzando hacia una noción de intelectualidad indígena que incluya no solo a los sujetos sino a los saberes. La presencia de estos proyectos educativos diferentes, no sin problemas y contradicciones, ha potenciado no solo el crecimiento de la profesionalización indígena, sino el surgimiento de un grupo de mujeres indígenas licenciadas universitarias.

Como señala Oehmichen (1999), la elaboración de una propuesta educativa particular para la población indígena data de la década de 1930 y se enmarca en las políticas sociales que era necesario llevar al campo para ampliar la base social de apoyo. En este sentido, el sexenio presidencial de Lázaro Cárdenas 
(1934-1940) se caracterizó, entre otras reformas, por la ampliación del proyecto educativo nacional que venía gestándose desde la década anterior a su mandato. En dicho proyecto eran integrados por el pasado, principalmente de los pueblos mayas y nahuas prehispánicos; pasado definido por sus descubrimientos y adelantos científicos valorados por la historia y la ciencia occidental. De esta forma, la valoración de los pueblos se basó en su identificación como sobrevivientes de sabios prehispánicos, sin reconocer sus formas culturales presentes.

En 1921 se había creado ya la Secretaría de Educación Pública (SEP), con el objetivo de combatir el analfabetismo, fomentar la escuela rural, la educación media superior y editar libros para promover las artes (Dietz y Mateos Cortés, 2011). Inicialmente, la ampliación de la escuela reconocía a la población pobre y rural, pero no la definía como a sujetos étnicos. Así, y con la preocupación por ampliar la red de educación rural, se crearon las primarias rurales, las misiones culturales para indígenas (fundadas en 1923) y las escuelas normales rurales para la castellanización. Como señalan los autores, en estas políticas los indígenas eran para la SEP «sujetos carentes de cultura y racionalidad, incapaces de generar conocimiento» (Dietz y Mateos Cortés, 2011, p. 67). Los maestros se vuelven pieza clave en este proceso, al llegar a las zonas rurales e indígenas como agentes del Estado, con capacidad de abonar a la promesa de modernización nacional a través de la castellanización de todos. Desde este papel central, y reconocido por la SEP, se va evidenciando la necesidad de generar un grupo de profesionales que, para atender estas escuelas, maneje las lenguas indígenas y sus formas culturales, todo con la finalidad de facilitar los procesos de castellanización y de adquisición de prácticas modernas.

Mientras se expandía la red de educación rural por interés nacional, la respuesta de la población indígena ante la escuela era ambigua. Como señalan Dietz y Mateos Cortés (2011), para la SEP el lastre lingüístico de los niños indígenas sería el principal impedimento para la ampliación de la educación en zonas rurales. Ante este diagnóstico, y sobre la base de la estructura generada por las escuelas rurales regionales y las escuelas centrales agrícolas, se fundan las normales rurales para formar a jóvenes indígenas hablantes de las lenguas locales como maestros bilingües. Las escuelas bilingües se inician y expanden como política nacional para garantizar una mayor eficiencia en el proceso de castellanización. Este es el inicio de la formación de docentes bilingües, aunque su profesionalización se dará solamente en la década de 1970 (Dietz y Mateos Cortés, 2011).

Como señalan Korsbaek y Sámano Rentería (2007), el indigenismo posrevolucionario generó políticas integracionistas, asimilacionistas, paternalistas 
y asistencialistas específicas para la población indígena. La antropología tuvo un importante papel en esta construcción de la política nacional para indígenas, articulada principalmente por el Instituto Nacional Indigenista (INI)3. Desde su surgimiento en 1948, el INI ha sido un importante aliado de la SEP en la difusión del castellano y de la escuela bilingüe, siendo que en la década de 1960 llevó a cabo varias experiencias en las que convocó y formó a jóvenes del campo con primaria completa para culminar la secundaria en un proceso de formación como promotores bilingües4. Estos primeros promotores bilingües fueron después incorporados por la SEP con plazas de docentes de escuelas bilingües (Dietz y Mateos Cortés, 2011).

Es así como el proyecto indigenista, además de promover un proceso de mestizaje desde la dilución de lo indígena en lo mestizo, acabó por promover un proceso de diáspora de la inicial y escasa intelectualidad indígena (Dietz y Mateos Cortés, 2011). Ejemplo de esto es el mencionado proceso de formación de jóvenes indígenas llevado a cabo por el INI, cuya consecuencia fue que una parte importante de ellos fuese reclutada para desempeñarse como capacitadores y formadores del INI, lo que los concentró en los centros urbanos; generando que los pueblos indígenas y las comunidades rurales permanecieran alejadas de los centros de poder y toma de decisiones. Además, el impacto en la ampliación del nivel educativo de la población indígena no siguió el proceso esperado, siendo que hasta hoy el nivel educativo alcanzado por la población indígena mexicana es bastante menor que el promedio nacional ${ }^{5}$.

Como señala Oehmichen (1999), entre mediados de la década de 1980 y la de 1990, las políticas indigenistas, en el marco de importantes transformaciones políticas y económicas globales, asumen una perspectiva nueva, paradójicamente,

Comisión Nacional para el Desarrollo de los pueblos Indígenas (CDI), desde 2003.

Hasta hoy existe en la CDI (antes INI) la figura de los promotores culturales indígenas, quienes funcionan como becarios del organismo, mas no como trabajadores estables (salvo una pequeña minoría no necesariamente indígena). Los promotores culturales indígenas acceden a puestos de un año por concurso, para recibir una remuneración definida como beca, y son responsables por llegar a las comunidades representando a la CDI para la aplicación de algunos programas productivos vinculados con las artesanías, definición de derechos, trabajo con mujeres y violencia, infancia, etc. Para esto, los promotores y promotoras culturales indígenas reciben capacitaciones de la CDI y tienen derecho a acceder a talleres y cursos que el organismo ofrece. Puede verse con más detalle el trabajo de la CDI en la convocatoria a becarios 2017 en este link: http://www.gob.mx/cdi/documentos/convocatorias-2017

5 No se cuenta con estadísticas al respecto; sin embargo, Schmelkes (2008) sostiene que para el año 2003 en torno al 1\% de la matrícula mexicana de educación superior era indígena, frente a una población indígena que representa el $10 \%$ de la población nacional. 
basada también en el ideal de modernización. Sostiene la autora que «ahora bajo métodos participativos» (1999, p. 10) se pretende la capacitación no escolar de los indígenas para transformarlos en socios e inversores privados. Su modernización, con el avance de la castellanización, pasa ahora por la capitalización, no por la escolaridad. Sin embargo, esta participación - siempre planeada desde arriba, desde los no indígenas y desde el ideal de modernización y progreso- proponía, en coherencia con el relanzamiento del discurso del desarrollo de la década de 1980 (Gudynas, 2014), un «desarrollo autónomo y autogestivo» que, frente al retraimiento del Estado como regulador, apunta hacia el fin del cooperativismo/ colectivismo impulsado en la reforma agraria (Oehmichen, 1999). En pocas palabras, el discurso de la autonomía y autogestión ve su límite en las políticas de desarticulación del colectivismo y el apoyo al campo.

A pesar de los problemas señalados, resulta evidente que este proceso «habría promovido el surgimiento de una plataforma de indígenas profesionales, que luego de algunos años se emancipa de la tutela institucional y se convierte en influyentes representantes políticos» (Dietz y Mateos Cortés, 2011, p. 72). En este marco de existencia de una pequeña clase profesional indígena de varias generaciones, surgen al inicio del siglo XXI, en lo que algunos autores llaman el tercer momento del indigenismo, las universidades interculturales, en un proceso de respuesta gubernamental a las demandas que, desde la década de 1990, venían siendo levantadas por el neozapatismo y las organizaciones indígenas.

Estos tres momentos de las políticas educativas para pueblos indígenas y su profesionalización presentan características muy distintas. En este caso, han sido recapituladas para señalar que, si bien el proceso de construcción de una intelectualidad indígena, de una clase de profesionales indígenas, venía gestándose desde el fin de la revolución, esta intelectualidad estaba constituida dentro de los límites de una formación asimilacionista y negadora de los saberes y epistemologías de los pueblos. Es por este motivo que, sin desconocer la existencia previa de la clase de intelectuales indígenas, en este trabajo se entiende que las licenciadas indígenas egresadas de la política de universidades interculturales representan, sí, un nuevo tipo de sujeto, por su inclusión mayoritaria como mujeres y por las formas y propuestas político-pedagógicas de estos espacios.

Resulta importante mencionar que, en todos estos momentos del indigenismo, se han generado procesos de resistencia y contrarresistencia, conviviendo las intenciones gubernamentales, con las acciones de organizaciones e individuos indígenas que se incorporan a las políticas desde sus propios intereses. De la misma forma puede entenderse la traducción que desde el Estado se hace de las 
demandas sociales, como procesos de contrarresistencia. Con esto se quiere señalar que no es posible pensar que, durante el siglo XX, las políticas indigenistas solo resultaran en la modernización de los indígenas profesionalizados, acoplados a la visión modernizante y desarrollista de la sociedad, sino que se formaron también intelectuales con pensamiento propio. Frente a este acotamiento es importante señalar que la política de las universidades interculturales representa también estas resistencias y contrarresistencias. Puede verse como una política que pretende transformar la demanda social en una conveniencia estatal, pero donde la resistencia busca tomar la política oficial como espacio de construcción propia. El gran campo de oportunidad que esta política representa para estas construcciones propias, a diferencia de las políticas indigenistas precedentes, está en la consideración no solo de los sujetos indígenas en la política, sino de sus saberes $\mathrm{y}$ formas de saber, que son formas colectivas.

\section{LAS UNIVERSIDADES INTERCULTURALES Y LA PROPUESTA DE LA UVI:OTROSMODELOS,OTROSOBJETIVOS...OTRASPROFESIONALES}

Las universidades interculturales mexicanas (UI) ${ }^{6}$ surgen como respuesta institucional a las demandas de los movimientos indígenas (Ávila Pardo, 2009). Sin embargo, se trata de una respuesta que no respeta las bases de la demanda indígena claramente establecida en los acuerdos de San Andrés Larráinzar. El 1 de enero de 1994 se llevó a cabo el levantamiento del Ejército Zapatista de Liberación Nacional (EZLN) en Chiapas. El EZLN se levantaba en demanda por los derechos de los pueblos indígenas y de los pobres, ante una historia de negación y abuso

\footnotetext{
Entre el año 2003 y el año 2015 se crearon o reconocieron, por la CGEIB-SEP, doce UI localizadas en los estados de Chiapas (UNICH), México (UIEM), Guerrero (UIEG), Hidalgo (UICEH), Michoacán (UIIM), Nayarit, Puebla (UIEP), Quintana Roo (UIMQRoo), San Luis Potosí (UISLP), Sinaloa (UAIS), Tabasco (UIET) y Veracruz (UVI). Sin embargo, la UI de Nayarit no ha iniciado su funcionamiento hasta la fecha, por lo cual contamos once UI. Del mismo modo, es importante señalar que la UVI se crea al interior de una universidad estatal convencional: la Universidad Veracruzana. Finalmente, también es importante mencionar que la Universidad Autónoma Indígena de México, localizada en Sinaloa y hoy llamada Universidad Autónoma Indígena de Sinaloa, así como la de San Luis Potosí, existían con anterioridad a la creación de las UI, por lo que son incorporadas y reconocidas por la CGEIB, mas no creadas por ella. Para más detalles sobre la creación de las UI y la oferta de educación superior intercultural en México, consultar el texto de Hernández Loeza (2017) en este mismo número. Cabe señalar que ninguna de las UI cuenta con autonomía universitaria, dependiendo de los gobiernos de los estados.
} 
que alcanzaría puntos álgidos con el fin oficial del reparto agrario ${ }^{7}$ y la firma del TLCAN. En octubre de 1995 se iniciaron los diálogos de San Andrés, que congregaron a organizaciones indígenas y campesinas, intelectuales, religiosos, funcionarios y legisladores, para discutir los motivos del levantamiento zapatista; es decir, la inclusión de los pueblos indígenas. En estas reuniones participaron 308 invitados y asesores del EZLN y 188 representantes del gobierno federal ${ }^{8}$. Luego de la consulta del EZLN con las bases zapatistas, se firman el 16 de febrero de 1996 los Acuerdos de San Andrés.

En estos acuerdos, el gobierno federal se compromete a reconocer a los pueblos indígenas en la Constitución, ampliar la participación y representación política de los pueblos indígenas, garantizar el acceso pleno a la justicia, promover las manifestaciones culturales de los pueblos indígenas, asegurar la educación y capacitación que incluya y respete sus saberes tradicionales, garantizar las necesidades básicas e impulsar la producción y el empleo. Para lograr estos objetivos se establece la necesidad de construir un nuevo marco jurídico que permitiese garantizar los derechos políticos, fortaleciendo su representación política y sus derechos jurisdiccionales, aceptando sus propios procedimientos para designar autoridades y sus sistemas normativos y sus derechos sociales, económicos y culturales, y respetando sus formas de organización, productivas y sus identidades ${ }^{9}$.

Los acuerdos y compromisos firmados en San Andrés establecen la necesidad de estas transformaciones jurídicas, constitucionales, estatales y sectoriales, señalando que toda reforma sobre los derechos indígenas sería consultada con el EZLN y el Congreso Nacional Indígena (CNI). En diciembre del mismo año se elabora la llamada Ley Cocopa, planteada por la Comisión de Concordia y Pacificación (Cocopa), que retoma los puntos centrales de los Acuerdos de San Andrés: reconoce el derecho a la autonomía incluyente de los pueblos indígenas, señala que los pueblos indígenas deben ser tomados en cuenta en la toma de decisiones que los afecten y afirma que se debe respetar y promover su cultura y su participación política, con una voz escuchada y atendida, con derecho a representaciones en el Congreso de la Unión y en los congresos de los estados.

Con la modificación d 1, artículo 27 constitucional en 1992.

Para una descripción detallada de la cronología de los acuerdos de San Andrés, la Ley Cocopa y la Ley indígena, puede consultarse el Centro de Documentación sobre el Zapatismo: http:// www.cedoz.org/site/content.php?doc $=405 \&$ cat $=6$ (consultado el 28 de septiembre de 2017)

$9 \quad$ El texto de los acuerdos de San Andrés y los tres documentos de propuestas y compromisos que lo acompañan están disponibles en: http://zedillo.presidencia.gob.mx/pages/chiapas/docs/ sanandres.html (consultado el 28 de septiembre de 2017). 
La Ley Cocopa nunca es sancionada y en su lugar se sanciona en 2001 la Ley Indígena, sin consulta a las organizaciones indígenas y sin la consideración de los Acuerdos de San Andrés. La Ley Indígena modifica los artículos 1, 2, 4, 8 y 115 de la Constitución Federal. El principal cambio está referido al carácter pluricultural de México y al establecimiento de la responsabilidad de la federación y los estados en garantizar educación pertinente (en contenidos y lenguas) en todos los niveles.

Como consecuencia, se crea en 2001, en la Secretaría de Educación Pública (SEP), la Coordinación General de Educación Intercultural y Bilingüe (CGEIB), desde donde se construye el modelo gubernamental para la creación de UI. Al igual que en la formulación de la Ley Indígena, las organizaciones indígenas no fueron consultadas para la creación del modelo de educación superior intercultural. Es ante este panorama que, para algunos autores, las UI representan la continuidad de las políticas indigenistas en lo que llaman «el neoindigenismo del siglo XXI» (Llanes Ortiz, 2008; Bastida Muñoz, 2005; Bello Domínguez, 2010). Desde esta comprensión, las propuestas gestadas por la Universidad de los Pueblos del Sur (UNISUR) en Guerrero y la Unitierra en Oaxaca representan, sin estar carentes de problemas, los espacios más importantes de construcción desde debajo, de procesos de inclusión de pueblos indígenas a la educación superior, desde la demanda articulada y organizada del movimiento indígena, campesino y afrodescendiente mexicano. De forma distinta, principalmente desde arriba, fueron pensadas y creadas las UI mexicanas en la primera década del siglo XXI. Como respuesta oficial ante la demanda social, las UI se construyen y controlan desde el poder estatal, lejos de las organizaciones y comunidades que atienden. A pesar de este distanciamiento del compromiso de participación, la política oficial de las universidades interculturales evidencia un giro importante con relación a las políticas que la preceden.

Como se señala en el libro Universidad intercultural. Modelo educativo (Casillas Muñoz y Santini Villar, 2009), estas instituciones de educación superior universitaria persiguen dos objetivos: ampliar la cobertura de educación universitaria, aproximándose a regiones rurales y de mayor concentración de población indígena en respuesta a su abandono histórico en las políticas educativas, y ofrecer un tipo de educación que parta del reconocimiento de la colonialidad del poder, característica del Estado nacional mexicano (y latinoamericano), que ha negado no solo la presencia física de la población indígena en la educación superior, sino también de sus saberes y formas de aprender. Como recuerdan Mateos Cortés y Dietz (2014), esta propuesta oficial de las UI se basa en la introducción de 
un enfoque intercultural que permita superar las estrategias asimilacionistas y homogeneizantes características del indigenismo del siglo XX. Sin duda, este segundo objetivo de las UI no es abrazado por todas ni por todos los gestores involucrados en su diseño e implementación; sin embargo, está en él el gran campo de oportunidades que ha permitido el surgimiento de espacios de debate internos a las UI donde ha sido posible cuestionar el modelo desarrollista y la educación a su servicio, para comenzar a crear, aun dentro de este modelo oficial, espacios de interculturalidad crítica.

Cabe señalar que esta confrontación con el desarrollismo no es lineal ni homogénea. Como recuerda Hernández Loeza ${ }^{10}$, algunas UI han tendido a abrazar el discurso del desarrollo como meta social, en la medida en que les ha permitido defender el componente de justicia social que persigue su surgimiento. Sin embargo, en la práctica, algunas UI, específicamente la Universidad Veracruzana Intercultural (UVI), se ven confrontadas con la necesidad de repensar el desarrollo, como consecuencia del diálogo de saberes que se contrapone a la visión unilineal del progreso. En las prácticas áulicas y extra áulicas, varios docentes de la UVI encuentran la necesidad de cuestionar que el desarrollo sea el camino para generar condiciones de vida digna para las comunidades desde una educación intercultural y diversa ${ }^{11}$. De esta forma, en las prácticas concretas y en la búsqueda por construir modelos educativos verdaderamente interculturales, la UVI se ha visto increpada por los límites de formar jóvenes profesionales funcionales a un sistema económico en crisis. Así, se ha visto tensionada por la confrontación con los ideales y principios del desarrollo que monopolizan los discursos sobre educación superior universitaria, que centralizan el incremento de egresos, la eficiencia terminal de los programas y la empleabilidad de sus egresados y egresadas, como los objetivos e indicadores de éxito de la educación superior. Frente a esto, su potencial no indigenista y emancipatorio está en la posibilidad de pensar otras formas de construir sociedad, formas capaces de no seguir los principios de la modernidad instrumental y del desarrollo (Quijano, 1988; Gudynas, 2014).

10 Comunicación personal, 20 de mayo de 2017.

11 Sobre esto vale la pena mencionar que, si por un lado algunos trabajos «externos» sobre la UVI han incorporado la categoría del buen vivir para el análisis, también desde dentro de la UVI algunos académicos y académicas han buscado incorporarla. Ejemplos de esto son el Festival del Buen Vivir organizado anualmente en la sede Grandes Montañas de la UVI, el Observatorio del Buen Vivir que se intenta construir en la sede Las Selvas de la UVI, y el trabajo de la comisión de planeación de la Nueva Oferta Educativa de la UVI que contempla la creación de la licenciatura en Agroecología y Buen Vivir. 
A diferencia de otras UI, la UVI surge por iniciativa de un grupo multidisciplinario de investigación, desde el Seminario de Educación Multicultural en Veracruz (SEMV), creado en 1996 dentro del Instituto de Investigaciones en Educación (IIE) de la Universidad Veracruzana (UV) (Ávila Pardo, 2009). En este panorama, en el año 2004 se firma un convenio entre el SEMV y la CGEIB para fundar la UVI como un programa al interior de la UV, la única UI reconocida por la SEP que no es una institución independiente (Ávila Pardo, 2009). Esta condición de la UVI como programa dentro de una gran universidad estatal ha implicado dificultades en la construcción de su propuesta intercultural, principalmente en términos de transformar procedimientos administrativos, pero su vinculación con los institutos y otros espacios académicos de la UVI le ha abierto un mayor abanico de espacios reflexivos.

En sus doce años de existencia, la relación UVI - UV ha pasado por distintos momentos de tensión y aproximación. Actualmente la UVI ha mostrado ser un campo importante para la UV en términos de la centralidad que vienen tomando la responsabilidad social universitaria, la sostenibilidad y la equidad de género, como nuevos criterios de evaluación de las universidades. Esto ha permitido un acercamiento que potencia la transversalización de la interculturalidad en toda la UV, además de mostrar que no se trata solo de abrir espacios para garantizar la presencia de mujeres y jóvenes indígenas, campesinos y afrodescendientes en la universidad, sino de generar espacios de diálogo de saberes apostando por la construcción de un modelo educativo no desarrollista.

Entendiendo el conocimiento como práctica, uno de los principios metodológicos que se defienden en la UVI, repetido por estudiantes y docentes, es contra el carácter extractivista de la investigación convencional ${ }^{12}$, una vez que la investigación propuesta por la UVI implica un enfoque inductivo y desde dentro, en trabajo colectivo con las organizaciones o comunidades a través de grupos de vinculación. El objetivo es garantizar una formación en el hacer de los y las jóvenes, desde el contacto con sus comunidades y que orienten sus aprendizajes al aporte en la construcción de propuestas que respondan a las demandas y necesidades de dichas colectividades. Para ello se ha generado un modelo de investigación vinculada para la gestión (IVG) propuesto en la UVI para maestros y estudiantes (Nava Vite, 2009).

12 Con investigación extractivista se hace referencia al proceso de recoger información e irse para producir documentos de lectura académica y de poco interés directo para los sujetos de la investigación. 
Desde la IVG, se entiende la investigación articulada al entorno y de forma concreta para la resolución de problemas sociales sentidos e identificados por los colectivos implicados ${ }^{13}$. Se rechaza la visión extensionista de la educación superior (la noción de extensión universitaria), para centralizar el diálogo y trabajo colectivo en la forma de la vinculación. De esta forma, la IVG no es solo la propuesta de investigación como uno de los campos de trabajo de la universidad, sino un eje transversal y central en el proceso discente y docente de la UVI ${ }^{14}$, permitiendo la formación de jóvenes investigadores que articulan la manera de entender la investigación y producción de conocimiento con la vinculación comunitaria en procesos participativos y de colaboración, además de centralizar la investigación-acción, dado que la gestión implica la acción para el bien colectivo.

En este marco la docencia no puede ser entendida separada de la investigación vinculada, como el aprendizaje no puede ser comprendido como meramente áulico. De igual forma, la investigación no puede comprenderse sin objetivos de impacto en la gestión de conflictos y problemas a través de la vinculación comunitaria. La IVG define, por lo tanto, también un perfil docente que es necesariamente investigador, gestor y vinculador, y perfiles específicos de estudiantes no solo de egreso, sino de ingreso, centralizando los vínculos comunitarios y compromiso social como características deseables en los postulantes ${ }^{15}$.

Con respecto al perfil de egreso, se espera que la UVI aporte a la construcción de sujetos que se reconozcan parte de colectividades y que desde este sentido de pertenencia posicionen sus aprendizajes al servicio del bien colectivo y sostenible ${ }^{16}$. Este objetivo implica una formación donde el saber hacer y la reflexión son los elementos más importantes y son posibles a partir del trabajo de IVG (Nava Vite, 2009). En los últimos años, además, desde la comprensión de las lenguas no como saberes sino como procesos epistémicos, se ha procedido a integrarlas al proceso formativo, más allá de su enseñanza-aprendizaje. Como parte de esto, se

13 Sobre la propuesta de IVG de la UVI ver: https://www.uv.mx/uvi/presentacion-del-area-deinvestigacion/

14 Puede consultarse el documento de la licenciatura ofrecida por la UVI (LGID) aquí: https:// www.uv.mx/uvi/files/2012/11/2007_LGID_DEx.pdf

15 En los documentos requeridos para e- ingreso se solicitan dos cartas: una de recomendación de autoridades comunitarias y otra de exposición de motivos. Se espera que ambos documentos evidencien el vínculo comunitario del postulante con su comunidad o una organización local. Los requisitos pueden verse en: https:/www.uv.mx/uvi/requisitos-para-licenciatura-en-gestionintercultural-para-el-desarollo/

16 Puede verse el perfil de egreso, así como la misión, visión y objetivos de la Licenciatura en Gestión para el Desarrollo en: https://www.uv.mx/uvi/licenciatura-en-gestion-intercultural-para-el-desarrollo/nuestros-egresados/ (consultado el 5 de septiembre de 2017). 
han defendido ya en todas las sedes trabajos recepcionales escritos en náhuatl y su presencia en aula, y en general en las dinámicas escolares, es cada vez mayor. Esto ha obligado no solo a defender la incorporación de docentes hablantes de náhuatl, sino también a instar a los docentes hispanohablantes a aprenderlo e incorporarlo a su práctica ${ }^{17}$.

La apuesta por el autoempleo es otro elemento central de la propuesta de interculturalidad crítica de la UVI. Las UI - y la UVI en específico- comprendieron desde su formación que se confrontarían con una crisis de modelo económico, y el consecuente desempleo, que cuestionaría la opción por ampliar la educación superior desde la lógica de la empleabilidad. Por este motivo, en contraposición a la propuesta neoliberal del emprendedurismo ${ }^{18}$, las UI proponen la formación de profesionales que se desempeñen como gestores comunitarios autónomos, capaces de gestionar recursos para llevar adelante iniciativas comunitarias ${ }^{19}$. Esto no siempre pasa por el asalariamiento o por el retorno económico, pero sí por la reactivación de formas de beneficio comunitario que implican prácticas de reciprocidad y contención colectiva, que van fortaleciendo procesos no monetarizados.

Todo esto marca distancia con el modelo universitario convencional, toda vez que la preocupación por la capacidad de generar nuevos espacios de empleo y ocupación en las comunidades para poner a su servicio los aprendizajes y reflexiones generados en la UVI marca una diferencia con el espíritu de la noción convencional de efecto educativo que centraliza la empleabilidad y asalariamiento para generar crecimiento económico (Olivera Rodríguez, 2015). Se trata, por lo tanto, de una propuesta distinta que va construyéndose, transformándose y fortaleciéndose como espacio descolonizador, intentando dejar atrás su condición de creación indigenista. Muchos pasos quedan pendientes, principalmente la mayor integración de las comunidades y organizaciones en el gobierno mismo de la universidad, que decide sobre el andar de la propuesta educativa; sin embargo, las potencialidades están cimentadas y pueden observarse, más allá de las propuestas e intenciones declaradas, en las reflexiones y trayectorias de licenciadas indígenas egresadas de la UVI.

17 A este respecto, el trabajo llevado a cabo por los docentes en la sede Grandes Montañas es particularmente exitoso.

18 Sobre los principios y propuestas del capitalismo en el neoliberalismo, pueden consultarse Shore y Wright (2000 y 2015) y Strathern (2000).

19 Sobre este punto puede consultarse también el artículo de Henández Loeza (2017) en este mismo número, donde se analiza lo acontecido en el primer encuentro de egresados de UI mexicanas, llevado a cabo en Xalapa en septiembre de 2015, organizado por la UVI. 


\section{LAS LICENCIADAS INDÍGENAS COMO ACTORAS EMERGENTES EN LA UVI}

En seis de las once UI reconocidas por la CGEIB en el período 2015-2016, la matrícula de mujeres fue mayor que la de hombres, y en la suma de la matrícula de las once en ese período, el $54 \%$ es de mujeres. Específicamente para el caso de la UVI, en el mismo período el $61 \%$ de la matrícula era femenina. Esto se ha explicado por la menor migración de mujeres y por la cercanía de la universidad a las comunidades, lo que posibilita que no deban dejar sus hogares para ir a estudiar. A pesar de esto, y aunque la matrícula se muestre con importante presencia femenina, la UVI encuentra dificultades particulares para apoyar a sus estudiantes mujeres y para ayudarlas a convencer a sus familias de «dejarlas» estudiar. Afirmaciones como «las mujeres no estudian, igual se van a casar y tendrán hijos» o «solo van a embarazarse a la escuela» no han desaparecido y son los principales argumentos para no permitir que las hijas mujeres vayan a la universidad. Además de esto, la carga de trabajo que tienen las jóvenes mujeres en sus casas hace que sea difícil para ellas visualizarse estudiando y a sus familias perdiendo ese trabajo cotidiano.

Al mismo tiempo, su presencia no es solo mayor en la universidad: su condición de permanencia en las localidades, frente a la migración masculina (frenada actualmente, pero muy fuerte en las zonas rurales de Veracruz en las últimas décadas), las evidencia como actoras comunitarias fuertemente articuladas y conocedoras de las dinámicas locales, lo que las coloca como estudiantes prometedoras en una propuesta educativa basada en la IVG. Sostengo, entonces, que frente a estos objetivos y modelo educativo de la UVI es necesario entender que lo que construyen las mujeres indígenas egresadas en su experiencia universitaria va más allá del incremento de posibilidades de empleabilidad, siendo que su articulación comunitaria se muestra potencializadora de dinámicas colectivas importantes para la resistencia al modelo económico. De esta forma, las mujeres indígenas no son solo mayoritarias en términos de la matrícula de la UVI, sino que tienen un papel importante en la ampliación y difusión de sus aprendizajes y diálogos de saberes en la comunidad.

La propuesta de la UVI y la emergencia de licenciadas indígenas egresadas del modelo intercultural representan el surgimiento de nuevas actoras que nos confrontan con la necesidad de ampliar la forma de entender el efecto de la experiencia universitaria. ¿Qué caracteriza entonces a estas actoras emergentes? ¿Cuáles son los aprendizajes y prácticas que las definen como gestoras interculturales para el beneficio comunitario? ¿Qué potenció el modelo intercultural de 
la UVI en sus egresadas? Para responder a estas preguntas, se describirán algunos aspectos de las experiencias como estudiantes y licenciadas de cinco egresadas de la primera generación de la UVI, sede Las Selvas.

El trabajo de campo y construcción de la información que alimenta estas reflexiones fue realizado en la localidad de Huazuntlán, municipio de Macayapan, en la sierra de Santa Marta, estado de Veracruz. El objetivo fue trabajar con entrevistas biográficas (Schriewer y Díaz Agea, 2015) que permitieran identificar no solo eventos y trayectorias, sino las valoraciones en torno a la universidad y su vinculación con otros resultados vivenciados como fruto de esa experiencia de escolaridad prolongada. Del total de diez egresadas mujeres de Huazuntlán de la primera generación (2005-2009), solo cinco aceptaron participar del estudio: Tonali, Alondra, Ana, Claudia y Natalia ${ }^{20}$. En el caso de Tonali, fue posible también realizar entrevistas biográficas con su madre, abuela y bisabuela, generando la posibilidad de hacer una comparación intergeneracional de cuatro niveles entre Tonali, egresada de la UVI; Ema, su madre con primaria incompleta; Matilde, su abuela con dos años de escolaridad y su bisabuela Elena, sin escolaridad e iletrada ${ }^{21}$. En este trabajo no se centralizará el contrapunto intergeneracional, pero es importante mencionarlo, porque es sobre la base de esta comparación que fue posible identificar la importancia de elementos como la transformación de patrones familiares.

De estas cinco egresadas, Alondra y Ana habían ingresado a la UVI con más de veintiocho años, siendo madres de familia y con trabajos adicionales a los de cuidado: Alondra costurera y Ana comerciante. Tonali ingresó a la UVI tres años después de haber concluido la educación media superior, cuando ya había descartado la opción de estudios universitarios por falta de recursos económicos. Solo Natalia y Claudia ingresaron a la UVI al año siguiente de concluir la preparatoria, y por lo señalado por ellas, de las diez egresadas de Huazuntlán de esa generación, solo serían tres las que ingresaron al año siguiente de terminar la educación media superior. Más claro en la primera generación que en los años siguientes, la UVI vino a absorber una demanda acumulada de educación superior, no solo por la proximidad, sino por el acceso a becas y por la consideración de las condiciones económicas, ocupacionales y familiares de los y las jóvenes de la región. También por esta acumulación inicial de la demanda es que la primera generación fue la más nutrida en todas las sedes y la que presentaba una media de edad más alta en los estudiantes. Sobre esto, Alondra comenta:

20 Seudónimos definidos con ellas para la difusión de sus reflexiones y experiencias.

21 Un análisis de la comparación intergeneracional es presentado en Olivera Rodríguez (2017). 
Cuando se anunció que llegaba la universidad acá, el pueblo entero fue a trabajar allá al terreno, y una compañera me dijo «yo voy a estudiar en la universidad», y yo dije «como quisiera, pero ya no puedo». Por lo económico, tenía yo dos hijos, uno en la prepa y otro en la secundaria. Ganas y capacidad no me faltan, «sé que puedo lograrlo», le dije a mi amiga. «¿Y entonces?», me dijo... Entonces pensé, y hablé con mi familia. Mis hijos me apoyaron, se alegraron por mí, pero mi esposo... No se opuso, pero dijo que no lo lograría y que no podía faltarle nada a la familia [...] No me resignaba, así que con apoyo de los maestros y de mi amiga y su esposo me fui a inscribir [...] Ya estando dentro, me dediqué completamente, sin límites, y el primer trimestre me promedio con 9. Y mi esposo me dijo «me ganaste, ya qué, continúa estudiando, se ve que sí puedes, échale ganas» (Alondra, nacida en 1972).

Aunque Alondra y Ana sostuvieron que como egresadas volvieron a trabajar en lo que hacían antes de ir a la UVI y no como licenciadas, reconocían también haber tenido experiencias como promotoras de programas de gobierno (desde encuestadoras en procesos muy específicos hasta becarias de CDI para la promoción de programas para población indígena) y particulares. Ana y Tonali tenían también experiencias constituyendo y participando en organizaciones para el trabajo asociativo con otras mujeres: Ana en una asociación de apicultores para la fabricación y venta de jabones y productos cosméticos naturales, y Tonali como parte de una asociación de jóvenes egresados de la UVI trabajando en la gestión de proyectos de financiamiento público.

No era solo la universidad, o sea lo que aprendíamos en la UVI, también los maestros nos empujaban siempre a hacer más cosas, nos contactaban con organizaciones, con cursos y diplomados que ofrecía el gobierno, o algunas asociaciones civiles. Ya egresadas también, también nos apoyaban. Me ayudaron esos cursos y contactos a fortalecer lo que veníamos haciendo en la asociación de apicultores de donde era mi papá [...] las esposas e hijas nos pusimos a preparar productos de la miel. Yo dirigía mucho ese proceso, creo que por mi experiencia y lo que aprendí en la universidad [...]. A mí me ayudó lo que me impulsaban los maestros y lo que aprendí en la UVI (Ana, nacida en 1978).

Claudia y Natalia, las más jóvenes, tuvieron también experiencias de trabajo en el sector público al salir de la UVI: Natalia vinculada con el programa de educación preescolar indígena y Claudia como promotora/becaria de la Comisión para el Desarrollo de los Pueblos Indígenas (CDI). Natalia había descubierto en la educación un campo de interés; sin embargo, el hecho de no tener la formación en pedagogía le imposibilitó conseguir un contrato como docente en la escuela donde colaboraba. Como resultado, decidió estudiar pedagogía, y es lo que hacía 
en régimen semipresencial al momento de la entrevista. Para cuando fueron realizadas las entrevistas, solo tres de las cinco egresadas se encontraban trabajando y asalariadas: Tonali, en una empresa privada de créditos; Natalia, en el sistema público de educación inicial, y Claudia, en CDI. Las tres coincidieron en la importancia que tuvo la UVI para su transformación. Esta transformación se define por diversos componentes: fortalecimiento de habilidades y competencias; cambios en las percepciones sobre sí mismas, sobre la familia y la comunidad, y ampliación y modificación de imaginarios y expectativas de futuro.

Por ejemplo, en las cinco entrevistas la centralidad del autorreconocimiento como indígenas fue un proceso que, aunque estando latente desde la infancia para algunas, fue aceptado y vivido con orgullo a partir de su paso por la universidad. En varios casos, incluso su reconocimiento como hablantes de náhuatl es percibido como un resultado del paso por la universidad.

Para mí fue importante, yo recuerdo que a mí me avergonzaba que me dijeran serrana. Así nos decían los de las comunidades de abajo porque somos serranos, de la sierra de Santa Marta. Pero en la escuela [UVI], aprendí que sí somos serranos porque venimos de la sierra y que eso no debe ser vergüenza, sino razón de orgullo. En mi caso, desde niña en mi casa hemos hablado el náhuatl y no hemos tenido vergüenza de eso, pero de ahí a decirnos indígenas igual y no. Tal vez era la palabra, nos decíamos nahuas, pero indígenas no... no se usaba la palabra. Y tenía compañeros y compañeras cuando empecé en la UVI que hasta mentían y decían que no sabían náhuatl, se avergonzaban. $\mathrm{Y}$ vi que todos cambiamos mucho, aprendimos, nuestros maestros eran excelentes maestros y nos criticaban esa vergüenza y nos mostraban el valor del náhuatl $[\ldots]$ Por eso también fue importante que la UVI estuviera aquí y que nos hicieran ver la importancia de revalorizar lo indígena, nuestra lengua, nuestros saberes. Yo aprendí mucho con esos maestros, sobre todo a aceptarme por lo que soy (Tonali, nacida en 1983).

Este autorreconocimiento se relaciona con el incremento de autoestima, al cual parece haber aportado también la experiencia formativa en la UVI desde la transformación de la visión de mujer y familia presente en la localidad. Al analizar las entrevistas, resultó evidente que, si bien las preocupaciones económicas y las posibilidades de ampliar el acceso a recursos monetarios eran centrales en las expectativas de vida de estas mujeres, un elemento recurrente, y especialmente subrayado por Tonali, es su definición como mujer profesional capaz de ser autosuficiente, sin necesidad de un esposo. Este campo de la autonomía es un importante diferencial en la construcción de vida cotidiana, si se compara la experiencia de Tonali con la de su madre, abuela y bisabuela. 
Yo veo mi vida, a mi familia, la que he construido, y me siento, la verdad, orgullosa. Porque no se parece en nada a la familia en la que yo crecí. Eso pues yo no me casé tan joven, aunque me embaracé cuando no lo esperaba y a partir de eso me junté, que sería como casarme [...] Pero era más grande que cuando se casaron mi mamá o mis abuelitas [...] creo que fue con menos de quince años. En cambio, yo ya tenía veinte años cuando me embaracé. Y Pancho era mi compañero de escuela, íbamos juntos en la UVI, yo lo elegí como novio, nadie arregló que me casara... Y, sí, nos tocó juntarnos y la pasamos difícil cuando tuvimos a Mateo, pero salimos adelante, salí adelante, terminé mis estudios y aprendí y crecí [...] Sobre todo, a veces cuando me siento cansada o molesta con él [Pancho], pienso que yo no dependo de él, que no lo necesito, porque si estuviera sola igual trabajaría, le echaría ganas y saldría adelante con mi hijo. Por eso, yo siento, yo sé que estoy con él porque quiero, no porque esté obligada o porque dependa de él (Tonali, nacida en 1983).

La experiencia de violencia familiar apareció en las conversaciones durante la investigación con las cinco egresadas entrevistadas, sus madres y otras mujeres de la localidad, como una experiencia constante y común a las familias de Huazuntlán. Esta violencia parecía vinculada con ciertos patrones matrimoniales presentes en las experiencias de las mujeres del estudio: matrimonios a muy temprana edad, con hombres mayores, arreglados por los padres y futuros suegros, donde la figura de la propiedad sobre ellas era permanente. Estas mujeres iniciaban pronto también la experiencia de la maternidad con la responsabilidad absoluta por el cuidado familiar, recluidas, a veces incluso físicamente, al ambiente de la casa. La comparación intergeneracional entre Tonali, su madre, abuela y bisabuela, permite interpretar que se trataba de una práctica expandida, y presente en las historias familiares de innumerables generaciones. Frente a esto, la transformación del imaginario de Tonali en mujer autónoma y profesional representa importantes cambios para su vida familiar y comunitaria.

Se trata de un impacto de la experiencia universitaria por la postergación de la maternidad y de la vida conyugal, por la definición de relaciones de pareja más parejas, pero además, por el reconocimiento del potencial que la profesionalización implica en términos emancipatórios. Resulta importante señalar que, si bien está presente una valoración del diploma universitario, del hecho de ser licenciada porque permite acceso a empleo asalariado, se está resaltando el valor de lo aprendido y crecido en el proceso educativo en la UVI. Es lo que señala Ana cuando indica que por lo aprendido pudo ser una líder en una asociación de apicultoras: se trata del reconocimiento que le dan sus compañeras por ser la universitaria, es decir, el valor del diploma o del grado, pero también por el 
valor de las competencias que se reconoce y se le reconocen. Por ejemplo, la capacidad de expresarse en público, de articular ideas de forma oral y escrita, de saber identificar y relacionarse con autoridades e instituciones privadas, la capacidad de pensar estrategias de trabajo, planes de acción y de gestionar apoyos, la capacidad e actualizarse y buscar información. Es también lo que dice Claudia:

Lo que yo veo es que somos profesionistas muy importantes, nos busca CDI, INEGI [ ...] pero no quieren valorar lo que sabemos. En CDI, nos ofrecen una beca para ser promotoras de proyectos para pueblos indígenas, proyectos de artesanías, o salud comunitaria, o de trabajo de niños y crianza... Pero ese trabajo, en realidad, lo que te pagan, la beca que le dicen, es muy poco, y en verdad no se necesita tener universidad. Lo que pasa es que CDI nos prioriza, nos llaman, a mí me llamaron para invitarme. Porque saben que conocemos la región, que sabemos la lengua, que podemos comunicarnos con las señoras, que nos conocen las autoridades y que tenemos compromiso y... bueno pues, todo lo que aprendimos en la escuela, de hacer diagnósticos, la vinculación, el trabajo con las autoridades [...] Saben que nos necesitan, aunque no nos paguen por eso (Claudia, nacida en 1987).

Vinculando los efectos de la experiencia universitaria señalados por Tonali, Claudia y las otras entrevistadas, con la propuesta educativa de la UVI, es posible identificar que la IVG representa el componente más importante como campo de construcción de habilidades y competencias (Constantino Toto, 2009 y Nava Vite, 2009). Esto, por ser campo de construcción de saberes-haceres (Mateos Cortés, Dietz y Mendoza Zuany, 2016), donde la lengua más que un saber es un proceso epistémico y donde las dinámicas comunitarias y la investigación acción son centrales. Se trata de la construcción de habilidades y competencias que combinan los requerimientos del trabajo intelectual y académico con los de expresión oral y escrita, de manejo y dirección de procesos de investigación, de análisis e interpretación de información y de articulación y difusión de propuestas de intervención, y con los requerimientos del trabajo comunitario que entiende la expresión oral y escrita como necesaria en diversas lenguas y en registros académicos y sociales, la investigación desde la acción participativa que implique la colaboración de los sujetos comunitarios y su inclusión transversal en los procesos de investigación (de la formulación a la interpretación), y la intervención con objetivos trazados desde visiones más complejas que la del desarrollo, para el beneficio comunitario y crítico del sistema económico desde la evidencia de sus límites. Como explican Claudia y Natalia: 
Nos buscan por eso, eso es lo que aprecian de nuestra formación. Saben que sus proyectos [de CDI] no son de trabajo de escritorio y quienes no saben hacer y no conocen el trabajo comunitario no obtienen buenos resultados [...] Porque tampoco es suficiente saber hablar en náhuatl para poder llegar con las señoras, con los señores, con los niños... hace falta que te conozcan. Tal vez no personalmente, pero que sepan de dónde vienes, quiénes son tu familia, tu localidad, y desde esa confianza, se comienza el diálogo, la comunicación. Muchos proyectos funcionan más porque cuando vamos, creen en nosotras, no es que crean en CDI (Claudia, nacida en 1987).

Lo que nosotras sabemos hacer, lo que hemos aprendido, no es solo porque hablamos náhuatl, es porque sabemos trabajar con las personas. Nos enseñaron a escuchar y proponer desde eso que aprendemos con ellos, hacer propuestas y formas de trabajar llamando, invitando a las personas a participar. Eso, yo he descubierto que no lo saben hacer muchos, algunos ni lo entienden... y después no saben por qué sus trabajos no funcionan (Natalia, nacida en 1988).

De esta forma, los elementos mencionados por Claudia y Natalia en torno al saber-hacer en el trabajo comunitario; el liderazgo entre compañeras señalado por Ana; las competencias que permiten salir adelante, como indica Alondra, y la construcción de autonomía señalada por Tonali, se convierten en efectos de la experiencia universitaria en la UVI. La ampliación del universo simbólico y de las posibilidades de construcción de proyectos individuales, familiares y colectivos, resulta también central. Estos nuevos proyectos pasan por el imaginario de otras dinámicas familiares, sin violencia y con mayor diálogo; de otros futuros laborales, como la continuación de los estudios a niveles de posgrado, $\mathrm{y}$ de combinar el trabajo comunitario — incluso de cuidado en la localidad - , con el apoyo en la investigación universitaria, papel que ha desempeñado Tonali en más de una ocasión asistiendo a una investigadora de la UV.

\section{NUEVAS ACTORAS}

Desde una visión crítica a la centralidad del mundo productivo y público que en la modernidad tendió a invisibilizar la importancia de la familia, la comunidad y el hogar como campos de construcción social político-afectivo (Assis Clímaco, 2016), estas transformaciones, aparentemente del orden de lo privado, tienen enorme importancia para pensar la reproducción de la vida individual, familiar y comunitaria. La identidad de licenciadas e indígenas de estas egresadas es, 
evidentemente, producto del paso por la UVI, no solo por ser licenciadas al haber cumplido el plan de estudios, sino por sus efectos en el autoestima, la visión sobre sí mismas, la postergación del matrimonio, la maternidad y el protagonismo en la definición de la pareja y de las pautas de la relación que se construye, la conciencia y reconocimiento del valor de las habilidades y competencias adquiridas en el perfil específico que la UVI propone, el sentimiento de autonomía que les permite imaginar futuros libres, y su efecto en las familias y la infancia como madres, abuelas, tías, vecinas, compañeras involucradas en el proceso de construir comunidad sin violencia.

Resulta central lo comentado por Claudia y Natalia: que la conciencia de estas egresadas sobre el valor de su perfil profesional no se haya visto acompañada por la valoración de dicho perfil en el mercado laboral. Según lo que señalan las egresadas entrevistadas — que se ha visto en espacios tales como el encuentro de egresados de UI de 2015-, resulta evidente que las instituciones públicas valoran el perfil de los egresados de las UI. Instancias como educación preescolar y primaria, CDI, Inegi, Inali y centros de salud, buscan y valoran los conocimientos y competencias de egresados y egresadas de la educación superior intercultural. Sin embargo, no los han incorporado al imaginario institucional de perfil profesional. Por este motivo, se les ofrecen empleos temporales muy puntuales como en el Inegi, o becas como en CDI.

Tonali, como otras licenciadas indígenas egresadas de la UVI, representa un nuevo tipo de profesional vinculada con las comunidades, que reflexiona desde las dificultades de ser mujeres en un mundo de tradiciones construidas por el desarrollo y la modernización que erigieron papeles subalternos para ellas, y que subvierten el orden de las cosas dando muestras claras de que el efecto educativo de la formación en la UVI, de interculturalidad crítica, no se reduce a la empleabilidad.

\section{REFERENCIAS BIBLIOGRÁFICAS}

Assis Clímaco, D. (2016). Ciencia en práctica. La emancipación desde las mujeres indigenas. Tesis de doctorado. Programa de Estudios Latinoamericanos, UNAM.

Ávila Pardo, A. (2009). Una historia para ser contada: orígenes de la UVI. En G. Alatorre Frenk (coord.), Un modelo educativo para la diversidad: la experiencia de la Universidad Veracruzana Intercultural. Xalapa, Veracruz: Universidad Veracruzana. 
Bastida Muñoz, M.C. (2005). Hacia la interculturalidad en las instituciones de educación superior. En M.A. Rodríguez (comp.), Foro de Educación, Ciudadanía e Interculturalidad (pp. 283-288). México: CGEIB - OCE - FLAPE - Contracorriente A.C.

Bello Domínguez, J. (2010). De la Educación Bilingüe Bicultural a las Universidades Interculturales en México. En E.A. Sandoval Forero, E. Guerra García y R. Contreras Soto (eds.), Políticas públicas de educación superior intercultural y experiencias de diseños educativos (pp. 60-87). Málaga: Universidad de Málaga.

Carot, J.M., C.R. Castro y M.M. Ojeda (coords.) (2013). Sistemas de información, indicadores y gestión universitaria. Rankings, transparencia y colaboración internacional. Xalapa, Veracruz, México [España]: Universidad Veracruzana: Imaginarial Editores: Universitat Politecnica de Valencia.

Casillas Muñoz, L. y L. Santini Villar (2006). Universidad Intercultural. Modelo educativo. México D.F: Secretaría de Educación Pública, Coordinación General de Educación Intercultural y Bilingüe.

Constantino Toto, M.C. (coord.) (2009). Libro Blanco DUVI. Veracruz: Universidad Veracruzana Intercultural.

Dietz, G. y L.S. Mateos Cortés (2011). Interculturalidad y educación en México. Un análisis de los discursos nacionales e internacionales en su impacto en los modelos educativos mexicanos. México: Secretaría de Educación Pública. Coordinación General de Educación Intercultural y Bilingüe.

González Apodaca, E. (2008). Los profesionistas indios en la educación intercultural. Etnicidad, intermediación y escuela en el territorio mixe. México: Universidad Autónoma Metropolitana y Casa Juan Pablos Centros Cultural.

Gudynas, E. (2014). El postdesarrollo como crítica y el buen vivir como alternativa. En G.C. Delgado Ramos (coord.), Buena vida, buen vivir: imaginarios alternativos para el bien común de la humanidad (pp. 61-95). México: CEIICH - UNAM.

Korsbaek, L. y M.A. Sámano Rentería (2007). El indigenismo en México: antecedentes y actualidad. Ra Ximhai, enero-abril, 3(1), 195-224. Universidad Autónoma Indígena de México, Mochicahui.

Llanes Ortíz, G.J. (2008). Interculturalización fallida: desarrollismo, neoindigenismo y universidad intercultural en Yucatán, México. TRACE, 53, 49-63.

Mateos Cortés, L. y G. Dietz (2014). Las universidades interculturales en México: un balance bibliográfico En S. Martí i Puig y G. Dietz (coord.), Empoderamiento y educación superior en contextos interculturales en México (pp. 41-62). Barcelona: Bellaterra.

Mateos Cortés, L., G. Dietz y G. Mendoza Zuany (2016). ¿Saberes-haceres interculturales? Experiencias profesionales y comunitarias de egresados de la educación 
superior intercultural veracruzana. Revista Mexicana de Investigación Educativa, 21(70), 809-835.

Muruyama, C. (2009). La economía política de la educación superior en México. México DF: Asociación Nacional de Universidades e Instituciones de la Educación Superior.

Nava Vite, R. (2009). Alcances y límites en la docencia e investigación vinculada: una experiencia desde la Huasteca. En G. Alatorre Frenk (coord.) Un modelo educativo para la diversidad: la experiencia de la Universidad Veracruzana Intercultural (pp. 151-162). Xalapa, Veracruz: Editorial Universidad Veracruzana.

Oehmichen, C. (1999). Reforma del Estado. Política social e indigenismo en México (1988-1996). México: Instituto de Investigaciones Antropológicas - UNAM

Olivera Rodríguez, I. (2015). ¿Desarrollo o bien vivir? Repensando la función social de la Universidad Intercultural desde el cuestionamiento al efecto educativo. Anthropologica, 32(33), 179-207.

Olivera Rodríguez, I. (2017). Las potencialidades del proyecto educativo de la Universidad Veracruzana Intercultural: una crítica al desarrollo desde la noción del buen vivir. Revista de la Educación Superior, 46(181), 19-35. https://doi. org/10.1016/j.resu.2016.08.004

Quijano, A. (1988). Lo público y lo privado: Un enfoque latinoamericano. En A. Quijano, Modernidad: identidad y utopía en América Latina. Lima: Sociedad y Política Ediciones.

Schmelkes, S. (2008) Creación y desarrollo inicial de las universidades interculturales en México: problemas, oportunidades y retos. En Daniel Mato (ed.), Diversidad cultural e interculturalidad en educación superior. Experiencias en América Latina (pp. 329-338). Caracas: Unesco.

Schriewer, K. y J.L. Díaz Agea (2015). Cuestiones prácticas en cuanto a la elaboración de relatos biográficos. Revista Miscel.lània, 20(1), 114-131.

Shore, C. y S. Wright (2015). Governing by numbers: audit culture, rankings and the new world order. Social Anthropology, 23(1), 22-28. https://doi.org/10.1111/14698676.12098

Strathern, M. (2000). Introduction: New accountabilities. Anthropological studies in audit, ethics and the academy. En M. Strathern (coord.), Audit cultures. Anthropological studies in accountability, ethics and the academy (pp. 1-18) Londres: Routledge. https://doi.org/10.4324/9780203449721 